\title{
Submitted: 19.06.2020 \\ Accepted: \\ A case of incidental infantile gallbladder adenomyomatosis: an unusual US finding of uncertain clinical significance
} 18.08.2020

Published: 18.12.2020

\section{Keywords}

adenomyomatosis, children, gallbladder

\author{
Elena Drakonaki1, Stamatios Kokkinakis², Ioannis Karageorgiou², \\ Neofytos Maliotis², Anna loannidoy ${ }^{3}$, Emmanouil K. Symvoulakis ${ }^{4}$ \\ ${ }^{1}$ Consultant Radiologist, Department of Anatomy, European University of Cyprus Medical \\ School, Nicosia, Cyprus \\ ${ }^{2}$ School of Medicine, University of Crete, Greece \\ ${ }^{3}$ Asklepeion General Clinic, Heraklion, Greece \\ ${ }^{4}$ Department of Social Medicine, School of Medicine, University of Crete, Greece \\ Correspondence: Elena Drakonaki, Diagnostic and Interventional Ultrasound Practice, \\ Daidaloy 30-32, 71112 Heraklion Crete Greece. Anatomy Department, Medical School \\ of the European University of Cyprus, Engomi, Nicosia, Cyprus; tel. +302810228300, \\ e-mail:drakonaki@yahoo.gr
}

DOI: $10.15557 / J o U .2020 .0055$

\begin{abstract}
Adenomyomatosis of the gallbladder is defined as hypertrophy of the gallbladder mucosal epithelium that invaginates into a thickened muscularis propria, leading to the formation of intramural diverticula. It is typically considered a benign condition most commonly affecting adults and, rarely, children. In this case report, we present a case of gallbladder adenomyomatosis in a 3-month-old infant. The diagnosis was made incidentally on ultrasound examination of the abdomen, in an otherwise asymptomatic child with no laboratory test abnormalities and no underlying disease. The purpose of this case report is to make infantile adenomyomatosis and its implications known to clinicians, as the literature on this topic is limited. To the best of our knowledge, this is the youngest reported case of adenomyomatosis in the pediatric population.
\end{abstract}

\section{Introduction}

Adenomyomatosis (ADM) of the gallbladder is considered to be a benign acquired abnormality of both the mucosa and muscularis layers of the gallbladder. The epithelium proliferates deeply into the hypertrophied muscularis propria, thus forming either embedded diverticula or sinus tracts known as the Rokitansky-Aschoff sinuses (RAS). The incidence of gallbladder ADM in adults has been reported in up to $9 \%$ of the gallbladder histopathological specimens following cholecystectomy ${ }^{(1,2)}$. Although it is a relatively common condition in adults, only a small number of cases occurring in childhood have been identified and reported. The most common complaint exhibited by adult patients is abdominal pain in the upper right quadrant, whereas in childhood the symptomatology includes unspecified abdominal pain in a non-specific location, vomiting and nausea ${ }^{(3-5)}$.
Ultrasonography (US) is the imaging gold standard for the diagnosis of ADM based on its characteristic appearance allowing on-the-spot diagnosis ${ }^{(6)}$. We present a case of an infant with ADM and gallbladder sludge without any predisposing factors, underlying diseases or symptoms identified on routine US.

\section{Case presentation}

A 3-month-old male infant was referred to our outpatient US department for an US examination of the abdomen. He was a full-term infant born by vaginal delivery, weighing $3.680 \mathrm{~kg}$, with no prenatal or perinatal complications. The infant had a few regurgitations of a small amount of gastric contents and mild irritability after feeding, indicating mild gastroesophageal reflux (GER). No other symptoms of severe GER were reported. The 


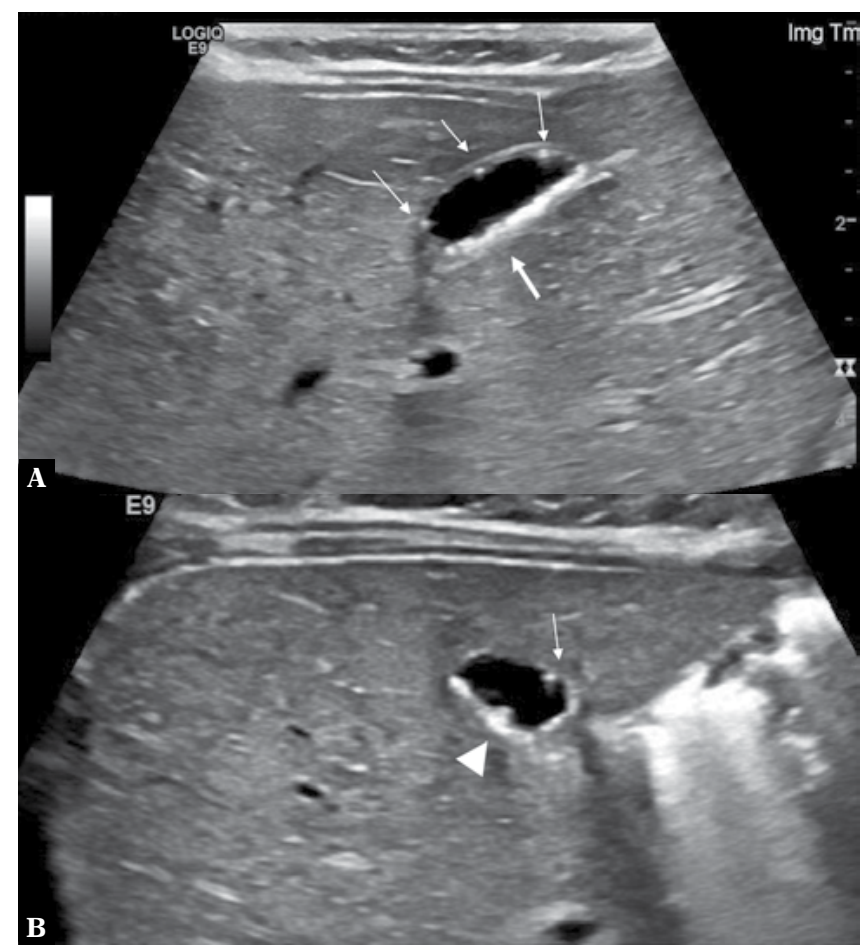

Fig. 1. Longitudinal (A) and axial (B) images of the gallbladder in a 3-month-old infant. There are multiple nondependent echogenic foci at the gallbladder wall with distal comet tail artifacts, in keeping with the typical Rokitansky-Aschoff sinuses (thin arrows). There is echogenic material in the dependent part of the gallbladder wall (thick arrow) that is not causing any acoustic shadowing, in keeping with bile sludge. There is a small accumulation of sludge in the wall protruding inside the gallbladder lumen, creating a polypoid projection (arrowhead)

physical examination was normal, and the infant was otherwise healthy and with normal developmental history. Although the symptoms were typical of GER, for which the diagnostic role of US is questionable, because of parents' anxiety, a US of the abdomen was scheduled. The US examination of the gastroesophageal area showed a normal intra-abdominal esophageal length with 2 short episodes of reflux within 10 minutes of scanning. No other abnormality was noted on US of the abdominal organs. Interestingly, the gallbladder had echogenic material without an acoustic shadow at the dependent surface, which was interpreted as sludge. To our surprise, the gallbladder wall was noted to have multiple (3-4) small echogenic foci with comet tail artifacts without any posterior shadowing, a typical US appearance of ADM (Fig. 1). Following this finding, complete laboratory blood tests were performed including aminotransferase and bilirubin levels, which were normal. Given the lack of clinical and laboratory signs of gallbladder or liver disease, the infant was released with instructions on the conservative management of GER. The US findings of the gallbladder were considered incidental and of doubtful clinical significance. On the follow-up after 3 months, the US appearance was unchanged, the laboratory tests were normal, and the infant remained in good health, while after 4 months, complete resolution of the aforementioned sonographic findings was noted (Fig. 2).

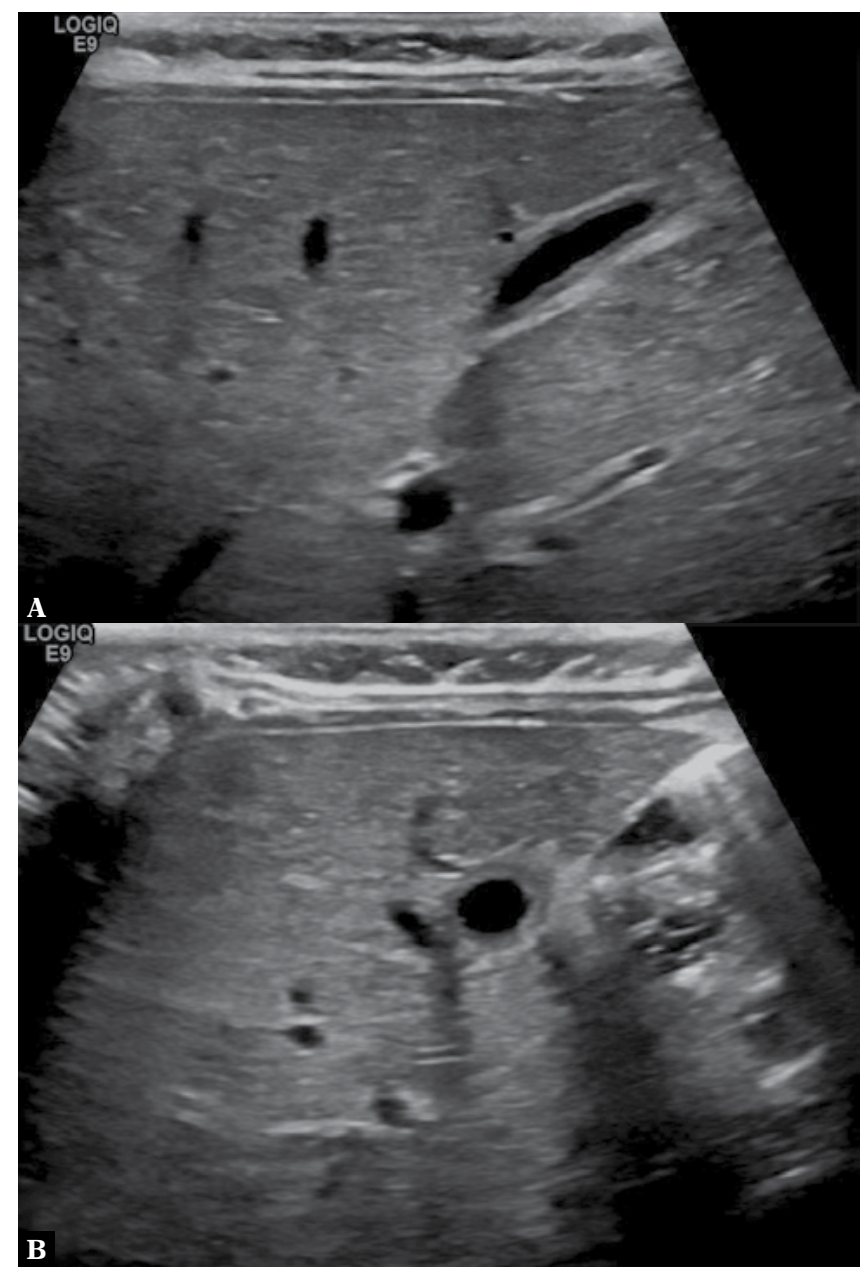

Fig. 2. Longitudinal (A) and axial (B) images of the gallbladder after one month, showing complete resolution of findings with return to the normal appearance of the gallbladder

\section{Discussion}

Gallbladder ADM is characterized by the proliferation of the mucosal epithelium extending into the thickened muscular layer of the gallbladder ${ }^{(2)}$. There are three types of ADM, including segmental, fundal, and a rarer diffuse form ${ }^{(2)}$. It is not yet clear what causes ADM, however, it is considered a degenerative disease rather than a congenital malformation, for there is a strong association with chronic gallbladder inflammation and cholelithiasis, which is present in more than half of the cases of adult $\mathrm{ADM}^{(2)}$. $\mathrm{ADM}$ is usually asymptomatic and a common finding in the histological examination of resected gallbladders (1-9\% of cholecystectomies), with the incidence increasing in patients older than 50 years $^{(2)}$. In the past, ADM was considered to predispose to malignancy, however the risk of gallbladder cancer in patients with ADM has not been clearly documented and understood ${ }^{(5)}$.

Preoperative imaging diagnosis of ADM is based on US, with the sensitivity reaching $65 \%$ due to the high resolution of the technique in imaging the gallbladder wall ${ }^{(2,5)}$. 
The US findings in ADM include increased wall thickness and echogenic or anechoic contents of the gallbladder's lumen such as sludge and calculi. However, the most characteristic US finding of gallbladder ADM are the Rokitansky-Aschoff sinuses (RAS) which are diverticula at the gallbladder wall forming a pseudo-cystic appearance ${ }^{(2)}$. Although RAS may also be present in xanthogranulomatous cholecystitis, they are necessary for establishing the diagnosis of $\mathrm{ADM}^{(2)}$. The presence of sludge inside RAS creates the characteristic reverberation artifact known as the "comet tail artifact" $(2,6)$. US is the gold standard and the preferred imaging modality for the diagnosis of ADM, with a similar accuracy to MRCP and a significantly higher accuracy than multi-detector $\mathrm{CT}^{(2,7)}$. ADM has not been associated with an increased risk of gallbladder adenocarcinoma by itself, but associated secondary changes such as cholelithiasis and cholecystitis may predispose to cancer $^{(8)}$.

Although gallbladder ADM is quite common and wellstudied in adults, it is very uncommon in the pediatric population. Only seven cases of gallbladder ADM have so far been reported in the literature ${ }^{(9-11)}$, in children ranging from 4 months to 11 years of age. The main complaint in those patients was nonspecific abdominal pain, and US was the first-line examination performed in all children, leading to the diagnosis of ADM. Six patients were treated surgically with cholecystectomy, whereas only one patient was treated conservatively with followup examinations, as ADM was an incidental finding in an otherwise asymptomatic infant. This patient is the only other case of gallbladder ADM reported in an infant who was also asymptomatic like our patient ${ }^{(10)}$. This patient, however, was on routine surveillance due to BeckwithWiedemann syndrome (BWS), which is not directly correlated to $\mathrm{ADM}^{(11)}$. To the best of our knowledge, our case is the youngest reported case of ADM in the literature, and it concerns an asymptomatic infant with no underlying pathology or laboratory test abnormality. As ADM may be completely asymptomatic and of no association to the underlying disease, it is possible that pediatric patients are not diagnosed until they present with abdominal pain due to sludge or gallstones later in life. Our case presented with the focal form of ADM with localized wall thickening with associated comet tail artifacts and the coexistence of biliary sludge.

\section{References}

1. Akçam M, Buyukyavuz I, Ciriş M, Eriş N: Adenomyomatosis of the gallbladder resembling honeycomb in a child. Eur J Pediatr 2008; 167: 1079-1081.

2. Golse N, Lewin M, Rode A, Sebagh M, Mabrut JY: Gallbladder adenomyomatosis: diagnosis and management. J Visc Surg 2017; 154: 345-353.

3. Ootani T, Shirai Y, Tsukada K, Muto T: Relationship between gallbladder carcinoma and the segmental type of adenomyomatosis of the gallbladder. Cancer 1992; 69: 2647-2652.

4. Aldridge MC, Bismuth H: Gallbladder cancer: the polyp-cancer sequence. Br J Surg 1990; 77: 363-364.

5. Alberti D, Callea F, Camoni G, Falchetti D, Rigamonti W, Caccia G: Adenomyomatosis of the gallbladder in childhood. J Pediatr Surg 1998; 33: 1411-1412.
Biliary sludge in infants and neonates is a benign finding of unclear clinical significance. Notably, gallbladder sludge can be identified antenatally via US in approximately $0.33 \%$ of fetuses. Most cases of gallbladder sludge tend to resolve within 6-12 weeks ${ }^{(12)}$, and the majority of patients are asymptomatic ${ }^{(13)}$. Interestingly, however, gallbladder sludge is also associated with multiple drugs and diseases. Examples mentioned in the literature include the presence of sludge in neonates and infants treated with ceftriaxone ${ }^{(14)}$, total parenteral nutrition ${ }^{(15)}$, and in patients with Down syndrome ${ }^{(16)}$, sickle cell disease $^{(17)}$, and congenital hypothyroidism ${ }^{(18)}$. However, our case did not have any of the above predisposing factors or diseases. The significance of biliary sludge and gallbladder wall thickening is not clear. In the pediatric population, isolated biliary sludge is neither associated with thickening of the gallbladder wall nor with acute cholecystitis, and thus is not an indication for cholecystectomy per $\mathrm{se}^{(19)}$. Our patient remained asymptomatic with a complete resolution of findings 4 months after the initial presentation.

\section{Conclusion}

In conclusion, ADM may appear incidentally on US examinations performed in infants and children, and may be completely asymptomatic. There is scarce evidence in the literature both about the incidence and the significance of this condition. Unlike in adult symptomatic patients, where ADM should be treated surgically, it seems that childhood ADM is treated conservatively and followed up using US examinations. However, as routine US is increasingly used in the evaluation of infants for minor indications, such as in our case, it is likely that the diagnosis rates of ADM in the pediatric population will increase, thus elucidating the possible clinical significance of this finding.

\section{Conflict of interest}

Authors do not report any financial or personal connections with other persons or organizations, which might negatively affect the contents of this publication and/or claim authorship rights to this publication.
6. Zani A, Pacilli M, Conforti A, Casati A, Bosco S, Cozzi DA: Adenomyomatosis of the gallbladder in childhood: report of a case and review of the literature. Pediatr Dev Pathol 2005; 8: 577-580.

7. Bang SH, Lee JY, Woo H, Joo I, Lee ES, Han JK et al.: Differentiating between adenomyomatosis and gallbladder cancer: revisiting a comparative study of high-resolution ultrasound, multidetector CT, and MR imaging. Korean J Radiol 2014; 15: 226-234.

8. Pang L, Zhang Y, Wang Y, Kong J: Pathogenesis of gallbladder adenomyomatosis and its relationship with early-stage gallbladder carcinoma: an overview. Braz J Med Biol Res 2018; 51: e7411.

9. Parolini F, Indolfi G, Magne MG, Salemme M, Cheli M, Boroni G et al.: Adenomyomatosis of the gallbladder in childhood: A systematic review 
of the literature and an additional case report. World J Clin Pediatr 2016; 5: 223-227.

10. Zarate YA, Bosanko KA, Jarasvaraparn C, Vengoechea J, McDonough EM: Description of the first case of adenomyomatosis of the gallbladder in an infant. Case Rep Pediatr 2014; 2014: 248369.

11. Eroğlu N, Erduran E, İmamoğlu M, Sağnak Z, Cansu A: Diffuse adenomyomatosis of the gallbladder in a child. J Pediatr Hematol Oncol 2016; 38: e307-e309.

12. Petrikovsky B, Klein V, Holsten N: Sludge in fetal gallbladder: natural history and neonatal outcome. Br J Radiol 1996; 69: 1017-1018.

13. Cancho Candela R, Díaz González J, Perandones Fernández C, Viñuela Rueda B, Relea Sarabia A, María Andrés de Llano J: [Echogenic material in fetal gallbladder: prenatal diagnosis and postnatal follow-up]. An Pediatr (Barc) 2004; 61: 326-329.

14. Ozturk A, Kaya M, Zeyrek D, Ozturk E, Kat N, Ziylan SZ: Ultrasonographic findings in ceftriaxone: associated biliary sludge and pseudolithiasis in children. Acta Radiol 2005; 46: 112-116.
15. Pang L, Zhang Y, Wang Y, Kong J: Pathogenesis of gallbladder adenomyomatosis and its relationship with early-stage gallbladder carcinoma: an overview. Braz J Med Biol Res 2018; 51: e7411.

16. Boëchat MC, da Silva KS, Llerena JC Jr, Boëchat PR: Cholelithiasis and biliary sludge in Downs syndrome patients. Sao Paulo Med J 2007; 125: 329-332.

17. Al-Salem AH, Qaisruddin S: The significance of biliary sludge in children with sickle cell disease. Pediatr Surg Int 1998; 13: 14-16.

18. Kurtoğlu S, Coban D, Akın MA, Akın L, Yıkılmaz A: Neonatal sludge: a finding of congenital hypothyroidism. J Clin Res Pediatr Endocrinol 2009; 1: 197-200.

19. Patriquin HB, DiPietro M, Barber FE, Teele RL: Sonography of thickened gallbladder wall: causes in children. AJR Am J Roentgenol 1983; 141: $57-60$ 